\title{
Inelastic neutron scattering study on boson peaks of imidazolium-based ionic liquids
}

\author{
Maiko Kofu ${ }^{\mathrm{a}}$, Yasuhiro Inamura ${ }^{\mathrm{a}, 1}$, Yosuke Moriya ${ }^{\mathrm{a}, 2}$, Andrey Podlesnyak ${ }^{\mathrm{b}}$, Georg Ehlers ${ }^{\mathrm{b}}$, Osamu \\ Yamamuro $^{\mathrm{a}, *}$ \\ ${ }^{a}$ Institute for Solid State Physics, University of Tokyo, Kashiwa, Chiba 277-8581, Japan \\ ${ }^{b}$ Quantum Condensed Matter Division, Oak Ridge National Laboratory, Oak Ridge, TN 37831, USA
}

\begin{abstract}
Low energy excitations of 1-alkyl-3-methylimidazolium ionic liquids (ILs) have been investigated by means of neutron spectroscopy. In the spectra of inelastic scattering, a broad excitation peak referred to as a "boson peak" appeared at 1-3 meV in all of the ILs measured. The intensity of the boson peak was enhanced at the $Q$ positions corresponding to the diffraction peaks, reflecting the in-phase vibrational nature of the boson peak. Furthermore the boson peak energy $\left(E_{\mathrm{BP}}\right)$ was insensitive to the length of the alkyl-chain but changed depending on the radius of the anion. From the correlation among $E_{\mathrm{BP}}$, the anion radius, and the glass transition temperature $T_{\mathrm{g}}$, we conclude that both $E_{\mathrm{BP}}$ and $T_{\mathrm{g}}$ in ILs are predominantly governed by the inter-ionic Coulomb interaction which is less influenced by the alkyl-chain length. We also found that the $E_{\mathrm{BP}}$ is proportional to the inverse square root of the molecular weight as observed in molecular glasses.
\end{abstract}

Keywords: ionic liquid, inelastic neutron scattering, boson peak, glass

2010 MSC: 00-01, 99-00

\section{Introduction}

Ionic liquide (ILs) are defined as salts in liquid states near room temperature. ILs have gained much attention in a past decade due to their unique properties, such as negligible vapor pressure, non-flammability, high ionic conductivity, and high solubility in a wide range of inorganic, organic, and polymeric materials.

5 It is remarkable that their physicochemical properties are tunable by varying cations and anions, which make them much more versatile and useful for applications. They consist of bulky and asymmetric organic cations and common anions. The cations of ILs have positively charged core parts and hydrophobic alkylchains. On the other hand, a wide range of anions are employed, ranging from halogens $\left(\mathrm{Cl}^{-}, \mathrm{Br}^{-}, \mathrm{I}^{-}\right)$ to inorganic/organic ionic groups (e.g. $\left.\mathrm{BF}_{4}^{-}, \mathrm{PF}_{6}^{-},\left(\mathrm{CF}_{3} \mathrm{SO}_{2}\right)_{2} \mathrm{~N}^{-}, \mathrm{CH}_{3} \mathrm{COO}^{-}\right)$. The most investigated ILs 10 are based on imidazolium cations, in particular, 1-alkyl-3-methylimidazolium salts which are investigated in this work. They are usually abbreviated as $\mathrm{Cn}$ mimX, where $n$ is the alkyl-carbon number and $\mathrm{X}$ is the anion.

Another interesting aspect is that some classes of ILs are "good" glass formers. The supercooled liquid undergoes a glass transition at the glass transition temperature, $T_{\mathrm{g}}$, which is typically around $200 \mathrm{~K}$ [1].

15 Beside the glass transition, there is an interesting dynamical phenomenon observed by inelastic neutron and Raman scatterings. In glasses and (sometimes) supercooled liquids, one can observe excess vibrational excitations over the Debye level, which is called "boson peak". The boson peak appears at typically 1$5 \mathrm{meV}$ irrespective of the type of chemical bonding. The peak intensities at different temperatures can be normalized with Bose factors in spite of intrinsic broadness of the peak shape, hence the name. The

\footnotetext{
*Corresponding author

${ }^{1}$ Present address:Neutron Science Section, J-PARC Center, Tokai, Ibaraki 319-1195, Japan

${ }^{2}$ Present address:School of Engineering, University of Tokyo, Bunkyo-ku, Tokyo 113-8656, Japan
} 
presence of the boson peak is universal for glass-forming materials and the origin of the boson peak is one of major subjects in glass researches. The question arises as to what kind of vibrational mode contributes to the boson peak. Recently, some spectroscopic and computer simulation studies suggested that the boson peak has a transverse character $[2-6]$ which can be related to the libration of molecules $[7,8]$ or structural fragments such as $\mathrm{SiO}_{4}$ tetrahedrons $[9,10]$. Furthermore it is discussed that the boson peak energy is 25 associated with the van Hove singularity [5] or Ioffe-Regel limit [3] for the transverse acoustic (TA) mode. However, the origin of the boson peak is still under debate [11-13].

As for the ILs, the boson peak has been investigated mainly by Raman spectroscopy [14-16]. Ribeiro et al. have investigated several types of ILs and found that the boson peak frequency is insensitive to the length of the alkyl-chain but depends on anions [16]. On the other hand, only $\mathrm{C} 4 \mathrm{mimCl}$ and $\mathrm{C}_{4} \mathrm{mimFeCl}_{4}$ were investigated by neutron scattering $[17,18]$. Neutron scattering have an advantage that the scattering intensity is directly related to the vibrational density of states, whereas for light scattering, a photon-phonon coupling constant, which is frequency dependent, makes it difficult to make a quantitative comparison of the boson peak among substances.

In the paper, we report our systematic studies of the boson peak for imidazolium-based ILs at glassy states, using an inelastic neutron scattering (INS) technique. We aim at investigating (i) how the feature of the boson peak changes with anions or cations in more detail and (ii) what kind of vibrational mode contributes to the boson peak in ILs. For the purpose, we have measured twelve ILs, eight protonated and four deuterated ones, comprising six anions and four cations with different alkyl-chain lengths. The scattering intensity of protonated ILs predominantly comes from incoherent scattering from $\mathrm{H}$ atoms, which

40 enables us to investigate the density of states. On the other hand, the deuterated samples will provide information for the collective dynamics. Although the boson peak usually appears as dispersionless mode in the $Q$ range studied in this work, it is of interest to see how the boson peak intensity varies as a function of momentum transfer $Q$.

\section{Experimental}

The cations chosen in this work are 1-alkyl-3-methylimidazolium ions (Cnmim, $n=3,4,6$, 8), while the anions are chloride $(\mathrm{Cl})$, bromide $(\mathrm{Br})$, iodide $(\mathrm{I})$, tetrafluoroborate $\left(\mathrm{BF}_{4}\right)$, hexafluorophosphate $\left(\mathrm{PF}_{6}\right)$, and bis(trifluoromethylsulfonyl)imide (TFSI) ions with a wide range of ionic size. The protonated $\mathrm{C} n \mathrm{minI}(n=3$, $4,6)$ were provided by Prof. M. Nakakoshi in Yokohama National University. All other protonated samples were purchased from commercial sources; $\mathrm{C} 4 \mathrm{mimX}\left(\mathrm{X}=\mathrm{Cl}, \mathrm{BF}_{4}, \mathrm{PF}_{6}\right.$, TFSI $)$ from Kanto Chemical Co., Inc. and $\mathrm{C} 4 \mathrm{mimBr}$ from Solvent Innovation $\mathrm{GmbH}$. The nominal purities of the samples were specified as $>99 \%$ and they were used without further purification. The deuterated samples were the same as used for the previous quasielastic neutron scattering studies and their synthesis methods are reported in Refs. [19, 20]. The samples were loaded into concentric double-cylinder Al cans with He gas, which facilitates thermal equilibration inside the cans. The thickness of the sample confined between the two $\mathrm{Al}$ walls was $0.3 \mathrm{~mm}$ for

55 the protonated samples and $2 \mathrm{~mm}$ for the deuterated analogues, giving a neutron transmission of 85-90\%, in order to reduce multiple scattering effects.

The INS experiments were performed using two time-of-flight spectrometers, the AGNES spectrometer [21], which is owned by ISSP, University of Tokyo and located at the research reactor JRR-3 in Japan Atomic Energy Agency, and the CNCS spectrometer [22] at the spallation neutron source SNS at Oak Ridge

60 National Laboratory in USA. All of the protonated samples were measured on AGNES. While the deuterated ones were investigated using CNCS, taking advantage of the high neutron flux at the SNS facility. The incident neutron energies used were $4.6 \mathrm{meV}$ at AGNES and $6.6 \mathrm{meV}$ at CNCS, giving rise to energy resolutions (full width at half maximum of the elastic peak) of $\Delta E=0.12 \mathrm{meV}$ and $\Delta E=0.2 \mathrm{meV}$, respectively. The deuterated C8mimTFSI was measured on CNCS also with the incident energy of $1.55 \mathrm{meV}$

${ }_{65}(\Delta E=0.025 \mathrm{meV})$. The data reduction was performed using an in-house software at AGNES and the Mantid software at CNCS. 


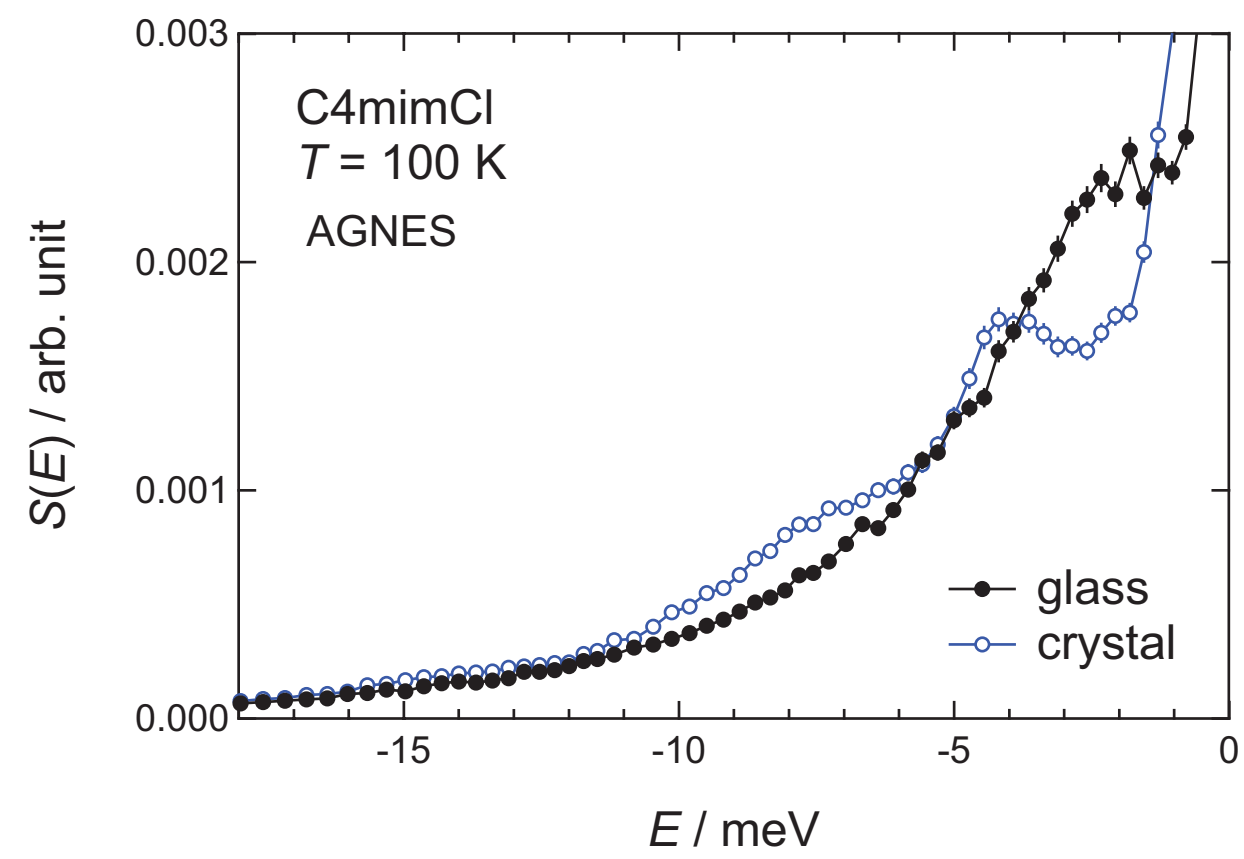

Figure 1: Energy dependence of scattering intensity averaged over $1<Q<2.5 \AA^{-1}$ for $\mathrm{C} 4 \mathrm{mimCl}$ at both glassy and crystalline states measured at $T=100 \mathrm{~K}$.

\section{Results and Discussion}

\subsection{Incoherent scattering}

Incoherent INS spectra were measured using the AGNES spectrometer for the protonated samples. Fig. 1 shows the energy spectra of inelastic scattering intensity, $S(E)$, of the glassy $\mathrm{C} 4 \mathrm{mimCl}$ at $T=100 \mathrm{~K}$. The polycrystalline $\mathrm{C} 4 \mathrm{mimCl}$, which was obtained by annealing the sample at $T=290 \mathrm{~K}$, is also presented for comparison. The present data are different from those previously reported in Ref. [18]; the AGNES instrument was upgraded in 2005 and the present data were measured after that. The plotted $S(E)$ values are estimated by summing up the intensity of the detectors with $Q$ values between 1 and $2.5 \AA^{-1}$ at elastic positions and then is normalized by the elastic intensity. Assuming that the scattering intensity comes from the incoherent scattering from $\mathrm{H}$ atoms, this normalization procedure allows us to compare $S(E)$ of the different samples quantitatively.

Apparently, the vibrational spectrum at the glassy state is quite different from that in the crystalline phase. The glassy $\mathrm{C} 4 \mathrm{mimCl}$ exhibits an excess vibrational excitation around $2 \mathrm{meV}$, demonstrating the presence of a boson peak. On the other hand, two vibrational peaks were observed at 4 and 8 meV in the crystalline phase. Penna and co-workers [16] observed Raman peaks around 3.8 and $10 \mathrm{meV}$ at $300 \mathrm{~K}$ but could not identify the origin of the peaks. Their peaks may be essentially the same as those observed by us. We speculate that the peak at $4 \mathrm{meV}$ arises mainly from the TA branch while the broad band at $8 \mathrm{meV}$ involve the contribution from the longitudinal acoustic (LA) mode and/or some optical phonons.

It is known that the boson peaks are more visible in a $g(E) / E^{2}$ plot. Here $g(E)$ is the vibrational density of states (VDOS) and $E^{2}$ expresses the Debye contribution. Here $G(E)$ was estimated from $G(E) \propto$ $S(E) \times E /\langle n\rangle$ in the incoherent and one-phonon approximations, where $\langle n\rangle$ is the Bose factor. Since a standard sample was not measured, we cannot obtain the absolute value of $g(E)$ and show it in an arbitrary unit. Fig. 2 displays the $g(E) / E^{2}$ for glassy $\mathrm{C} 4 \mathrm{mimX}\left(\mathrm{X}=\mathrm{Cl}, \mathrm{Br}, \mathrm{I}, \mathrm{BF}_{4}, \mathrm{PF}_{6}, \mathrm{TFSI}\right)$ taken at $T=100 \mathrm{~K}$. 
peak energy, the spectrum was fitted to the combination of a log-normal function, $1 / E$, and a constant,

$$
\frac{g(E)}{E^{2}}=I_{\mathrm{BP}} \exp \left[-\frac{\left\{\ln \left(E / E_{\mathrm{BP}}\right)\right\}^{2}}{\sigma^{2}}\right]+\frac{A}{E}+C .
$$

Here $E_{\mathrm{BP}}$ is the peak-top energy of the boson peak, $I_{\mathrm{BP}}$ is the intensity of the boson peak, $\sigma$ is associated with the peak width. The log-normal function has often been used to describe the boson peak [23-26]. The term $A / E$ is added to reproduce the tail near the elastic line. The constant expresses the contribution from

95 the Debye phonon. The solid curves are the results of the fits and dashed ones correspond to the individual components in Fig. 2. The $g(E) / E^{2}$ plot for CnminI $(n=3,4,6)$ at glassy state are presented in Fig. 3. The boson peak did not change much with changing the allyl-chain length. The same fitting procedure was carried out for these data. The width parameter $\sigma$ is about $1 \mathrm{meV}$ and unchanged for all of the measured samples.

\subsection{Coherent scattering}

Fig. 4 shows the $S(Q, E)_{\text {red }} / Q$ map of the inelastic neutron scattering from d-C8mimTFSI at $40 \mathrm{~K}$. Here $S(Q, E)_{\text {red }}$ is the reduced dynamic structure factor and given by $S(Q, E)_{\text {red }}(=S(Q, E) / E\langle n\rangle)$. The background including a spurious scattering due to multiple scattering events involving the cryostat and the sample was subtracted. Based on the principle of the neutron scattering, the scattering intensity of the vibrational excitations becomes stronger as $Q$ is increased. Hence the $S(Q, E)_{\text {red }} / Q$ contour map is often shown in order to make the vibrational excitations more visible. In the $S(Q, E)_{\text {red }} / Q$ map, the excitations in $E<0$ are almost the mirrored image of those in $E>0$. The dominant feature of the spectrum is that the vibrational excitations appear at roughly constant energy of $\pm 2 \mathrm{meV}$ with a modulation of the scattering intensity in $Q$; the scattering intensity is enhanced around $Q=1.5 \AA^{-1}$ and $3 \AA^{-1}$. It should be pointed out that the excitation appears to have no dispersion in
dispersionless nature is characteristic of the boson peak.

Fig. 5 presents the $g(E) / E^{2}$ plots for the deuterated ILs. The $g(E) / E^{2}$ values were estimated in the same manner as for the data of the protonated ILs (Figs. 2 and 3). The spectra were well fit to the same function as in the protonated ILs. The boson peak energy remains almost the same for d-C8mimCl and

$115 \mathrm{~d}-\mathrm{C} 4 \mathrm{mim} \mathrm{Cl}$. On the other hand, keeping the cation of d-C8mim, changing the anion $\mathrm{Cl}_{\text {by }} \mathrm{PF}_{6}$ or TFSI makes the boson peak energy shift to a lower energy side. The difference between the anion and cation effects on the boson peak energy is clearly seen as in the case of the protonated ILs.

It is worth examining the $Q$-dependence of the boson peak intensity. In Fig. 6, we compare the $Q$ dependence of the inelastic scattering intensity with that of the elastic one. The inelastic and elastic

120 intensities were evaluated by integrating $S(Q, E)$ over $-2.5 \leq E \leq-1 \mathrm{meV}$ and $-0.2 \leq E \leq 0.2 \mathrm{meV}$, respectively. The inelastic intensity is divided by $Q$ as in Fig. 4. The intensity modulation for both elastic and inelastic scattering is weaker in $\mathrm{d}-\mathrm{C} 8 \mathrm{mimCl}$, due to a weak contrast of scattering length, and so the data were not shown here. In fact, there exists the contribution from the incoherent scattering even for the deuterated samples, which is about 26 and $23 \%$ for the $\mathrm{PF}_{6}$ and TFSI samples, respectively. It slightly

125 smeared the intensity modulation in $Q$. It is apparent that the inelastic intensity exhibits clear peaks at the $Q$ positions of the second $\left(Q=1.5 \AA^{-1}\right)$ and third $\left(Q=3.0 \AA^{-1}\right)$ peaks in $S(Q, E=0)$. It indicates that the boson peak has a feature of the in-phase vibrational motion. The enhancement of $S(Q, E)$ at the first peak position $\left(Q=1.0 \AA^{-1}\right.$ for d-C8mimPF 6 and $0.8 \AA^{-1}$ for d-C8mimTFSI) is much weaker than those at the second and third peak positions, though conclusive discussion is difficult because the background near ${ }_{130} Q=0$ conceals the signals. Similar results have been reported for vitreous silica [9, 10, 27-30]. Buchenau et al. showed that the boson peak in vitreous silica is reproduced by a coupled rotation of five corner-shared $\mathrm{SiO}_{4}$ tetrahedra, by calculating the $Q$-dependence of the inelastic intensity [10]. It, however, is difficult to propose an explicit vibrational model for ILs with complex structures.

\subsection{Characteristics of Boson peak in ionic liquids}

135

We discuss what governs the boson peak energy $\left(E_{\mathrm{BP}}\right)$ of ILs. The $E_{\mathrm{BP}}$ values, which are obtained by fitting $G(E) / E^{2}$ to the log-normal function, are plotted against the anion radius $\left(r_{\text {anion }}\right)$ in Fig. 7 for all 
the samples measured. The $r_{\text {anion }}$ used here is the ionic radius given by Goldschmidt for anionic halogen and the value, evaluated from the Van der Waals volume reported by Ue et al. [31], for other anions. There is a clear tendency that $E_{\mathrm{BP}}$ shifts to a lower energy as $r_{\text {anion }}$ increases. On the other hand, $E_{\mathrm{BP}}$ remains almost unchanged for the three iodide ILs. Thus, $E_{\mathrm{BP}}$ does not depend on the length of the alkyl-chain if the anion is the same, but it depends on the anion size.

The variation of $E_{\mathrm{BP}}$ against $T_{\mathrm{g}}$ is presented in Fig. 8. The $T_{\mathrm{g}}$ values shown here were determined by differential scanning calorimetry for $\mathrm{C} \mathrm{mimBF}_{4}[32]$ and $\mathrm{C} 8 \mathrm{mimPF} \mathrm{F}_{6}[33,34]$ and by adiabatic calorimetry for other ILs $[17,19,35-39]$. Here we assume that the isotope effect is weak for $T_{\mathrm{g}}$. Fig. 8 shows that to the boson peak of ILs. In fact, in ILs, $T_{\mathrm{g}}$ becomes higher as $r_{\text {anion }}$ decreases but is insensitive to the length of alkyl-chain, which is analogous to the anion/cation dependency on the $E_{\mathrm{BP}}$. Consequently, the $E_{\mathrm{BP}}$ increases linearly with $T_{\mathrm{g}}$. Therefore, we conclude that both $E_{\mathrm{BP}}$ and $T_{\mathrm{g}}$ in ILs is mainly dominated by the inter-ionic Coulomb interaction. It is worth noting that the relation between $E_{\mathrm{BP}}$ and $T_{\mathrm{g}}$ is analogous for both $\mathrm{C} 4 \mathrm{mimX}$ and $\mathrm{C} 8 \mathrm{mimX}$. It means that the inter-ionic interaction is little influenced by the alkylchain. This is consistent with the fact that ILs exhibit nanoscale segregation of polar (core parts of cations and anions) and nonpolar (alkyl-chain) domains; the alkyl-chain does not penetrate into the polar domain, leading a small change in the inter-ionic distance. In other words, the length scale of the glass transition is shorter than that of the segregation and the molecules sense only local environment.

In Fig. 9, the $E_{\mathrm{BP}}$ values are plotted as a function of the inverse square root of molecular mass $\left(M^{-1 / 2}\right)$ along with those of previously studied molecular glass [40-42]. Here $M$ values of ILs are just a sum of $M_{\text {cation }}$ and $M_{\text {anion }}$, assuming the in-phase motion; the reduced mass $\left(M_{\text {cation }} M_{\text {anion }} /\left[M_{\text {cation }}+M_{\text {anion }}\right]\right)$ should be considered in the case of out-of-phase motions. Yamamuro et al. showed that the $E_{\mathrm{BP}}$ values lineally increases with $M^{-1 / 2}$ passing through the origin $(0,0)$ for several molecular glasses [42]. It implies that the boson peak of molecular glasses is like a harmonic oscillator. The $E_{\mathrm{BP}}$ values of the ILs also seems to lie on a straight line though the deviation is larger. It is pointed out that the slope of the plot for the ILs is larger than that for the molecular glasses. This fact suggests the stronger force constant for the ILs, presumably due to the inter-ionic Coulomb interaction. However, the nature of the correlation between $E_{\mathrm{BP}}$ and $M^{-1 / 2}$ still remains a puzzle; the question arises as to why the force constant looks constant for the glasses with the different masses. In order to understand the correlation, systematic studies for many types of glasses are required.

\section{Conclusions}

We have measured inelastic neutron scattering spectra of 1-alkyl-3-methylimidazolium ionic liquids (ILs) at glassy states. For all of the ILs measured, a broad excitation peak, called "boson peak" which is a

vibrational excitation characteristic of glasses, is observed around the energy of 1-3 meV. In the deuterated ILs where the coherent scattering is mainly captured, the intensity of the inelastic scattering is peaked at the $Q$ positions corresponding to the diffraction peaks. This indicates that the boson peak has a character of in-phase vibrational motion.

We found that the boson peak energy $\left(E_{\mathrm{BP}}\right)$ is insensitive to the length of alkyl-chain but decreases as 175 the anion size increases. Besides, the $E_{\mathrm{BP}}$ has a linear relationship with the $T_{\mathrm{g}}$. We thus conclude that both $E_{\mathrm{BP}}$ and $T_{\mathrm{g}}$ in ILs are determined mainly by the inter-ionic Coulomb interaction, which is hardly affected by the alkyl-chain. This is related to the fact that there exists the nanoscale segregation of polar/nonpolar entities in ILs. We also presented a linear relation between $E_{\mathrm{BP}}$ and the inverse square root of molecular mass. The slope for $E_{\mathrm{BP}}$ of the ILs is larger than that for the molecular glasses, suggesting the stronger

\section{Acknowledgement}

We thank Prof. M. Nakakoshi, Dr. T. Ueki, and Prof. M. Watanabe in Yokohama National University for the synthesis of the samples. We are deeply grateful to the Oak Ridge National Laboratory for giving 
us the opportunity to carry out our experiments when the Japan Proton Accelerator Research Complex

(J-PARC) was closed due to the earthquake in 2011. Research at ORNL's Spallation Neutron Source was sponsored by the Scientific User Facilities Division, Office of Basic Energy Sciences, U.S. Department of Energy. This work is financially supported by the US-Japan Cooperative Program on Neutron Scattering.

\section{References}

[1] S. Zhang, N. Sun, X. He, X. Lu, X. Zhang, J. Phys. Chem. Ref. Data 35 (2006) 1475-1517.

[2] G. Ruocco, F. Sette, J. Phys.: Condens. Matter 13 (2001) 9141-9164.

[3] H. Shintani, H. Tanaka, Nature Mater. 7 (2008) 870.

[4] A. Monaco, V.M. Giordano, Proc. Natl. Acad. Sci. 106 (2009) 3659.

[5] A. I. Chumakov, G. Monaco, A. Monaco, W. A. Crichton, A. Bosak, R. Rüffer, A. Meyer, F. Kargl, L. Comez, D. Fioretto, H. Giefers, S. Roitsch, G. Wortmann, M. H. Manghnani, A. Hushur, Q. Williams, J. Balogh, K. Parliński, P. Jochym, and

195 P. Piekarz, Phys. Rev. Lett. 106 (2011) 225501.

[7] O. Yamamuro, I. Tsukushi, T. Matsuo, K. Takeda, T. Kanaya, K. Kaji, J. Chem. Phys. 106 (1997) 2997.

[8] O. Yamamuro, K. Takeda, I. Tsukushi, T. Matsuo, Physica B 311 (2002) 84-89.

[9] U. Buchenau, N. Nücker, A. J. Dianoux, Phys. Rev. Lett. 53 (1984) 2316.

200 [10] U. Buchenau, M. Prager, N. Nücker, A. J. Dianoux, N. Ahmad, W. A. Phillips, Phys. Rev. B 34 (1986) 5665.

[11] B. Rufflié, G. Guimbretière, E. Courtens, R. Vacher, and G. Monaco, Phys. Rev. Lett. 96 (2006) 045502.

[12] M. T. Dove, M. J. Harris, A. C. Hannon, J. M. Parker, I. P. Swainson, M. Gambhir, Phys. Rev. Lett. 78 (1997) 1070

[13] G. Ruocco, A. Matic, T. Scopigno, S. N. Yannopoulos, Phys. Rev. Lett. 98 (2007) 079601.

[14] M. C. C. Ribeiro, J. Chem. Phys. 133 (2010) 024503.

205 [15] M. C. C. Ribeiro, J. Chem. Phys. 134 (2011) 244507.

[16] T. C. Penna, L. F. O. Faria, J. R. Matos, M. C. C. Ribeiro, J. Chem. Phys. 138 (2013) 104503.

[17] O. Yamamuro, Y. Minamimoto, Y. Inamura, S. Hayashi, H. Hamaguchi, Chem. Phys. Lett. 423 (2006) 371-375.

[18] O. Yamamuro, Y. Inamura, S. Hayashi, H. Hamaguchi, AIP Conf. Proc. 832 (2006) 73.

[19] O. Yamamuro, T. Yamada, M. Kofu, M. Nakakoshi, M. Nagao, J. Chem. Phys. 135 (2011) 054508.

${ }_{210}^{210}[20]$ M. Kofu, M. Nagao, T. Ueki, Y. Kitazawa, Y. Nakamura, S. Sawamura, M. Watanabe, O. Yamamuro, J. Phys. Chem. B 117 (2013) 2773-2781.

[21] T. Kajitani, K. Shibata, S. Ikeda, M. Kohgi, H. Yoshizawa, K. Nemoto, K. Suzuki, Physica. B 213-214 (1995) 872.

[22] G. Ehlers, A. A. Podlesnyak, J. L. Niedziela, E. B. Iverson, P. E. Sokol, Rev. Sci. Instrum. 82 (2011) 85108.

[23] I Pócsik, M. Koós, Solid State Commun. 74 (1990) 1253-1256.

215 [24] V. K. Malinovsky, V. N. Novikov, A. P. Sokolov, Phys. Lett. A 153 (1991) 63-66.

[25] L. Hong, B. Begen, A. Kisliuk, C. Alba-Simionesco, V. N. Novikov, A. P. Sokolov, Phys. Rev. B 78 (2008) 134201.

[26] Y. Matsuda, M. Kawashima, Y. Morita, T. Yamada, O. Yamamuro, S. Kojima, J. Phys. Soc. Jpn. 79 (2010) 033801.

[27] M. Arai, Y. Inamura, T. Otomo, N. Kitamura, S. M. Bennington, A. C. Hannon, Physica B 263-264 (1999) 268-272.

[28] M. Nakamura, M. Arai, T. Otomo, Y. Inamura, S. M. Bennington, J. Non-Cryst. Solids 293-295 (2001) 377-382.

220 [29] M. J. Harris, S. M. Bennington, M. T. Dove, J. M. Parker, Physica B 263-264 (1999) 357-360.

[30] M. J. Harris, M. T. Dove, J. M. Parker, Mineral. Mag. 64 (2000) 435-440.

[31] M. Ue, A. Murakami, S. Nakamura, J. Electro. Soc. 149 (2002) A1385-A1388.

[32] H. Tokuda, S. Tsuzuki, Md. A. B. H. Susan, K. Hayamizu, M. Watanabe, J. Phys. Chem. B 110 (2006) 19593-19600.

[33] S. Chun, S. V. Dzyuba, R. A. Bartsch, Anal. Chem. 73 (2001) 3737-3741.

225 [34] F. Nemoto, M. Kofu, O. Yamamuro, J. Phys. Chem. B submitted.

[35] Y. U. Paulechka, G. J. Kabo, A. V. Blokhin, A. S. Shaplov, E. I. Lozinskaya, Ya. S. Vygodskii, J. Chem. Thermo. 39 (2007) 158-166.

[36] G. J. Kabo, A. V. Blokhin, Y. U. Paulechka, A. G. Kabo, M. P. Shymanovich, J. Chem. Eng. Data 49 (2004), 453-461.

[37] A. V. Blokhin, Y. U. Paulechka, A. A. Strechan, G. J. Kabo, J. Phys. Chem. B 112 (2008) 4357-4364.

230 [38] Y. U. Paulechka, A. V. Blokhin, G. J. Kabo, A. A. Strechan, J. Chem. Thermo. 39 (2007) 866-877.

[39] O. Yamamuro et al. in preparation

[40] J. Wuttke, M. Kiebel, E. Bartsch, F. Fujara, W. Petry, H. Sillescu, Z. Phys. B91 (1993) 357.

[41] B. Frick, J. Williams, S. Trevino, R. Erwin, Physica B 213-214 (1995) 506.

[42] O. Yamamuro, I. Tsukushi, T. Matsuo, K. Takeda, T. Kanaya, K. Kaji, Prog. Theor. Phys. suppl. 126 (1997) 93-96. 


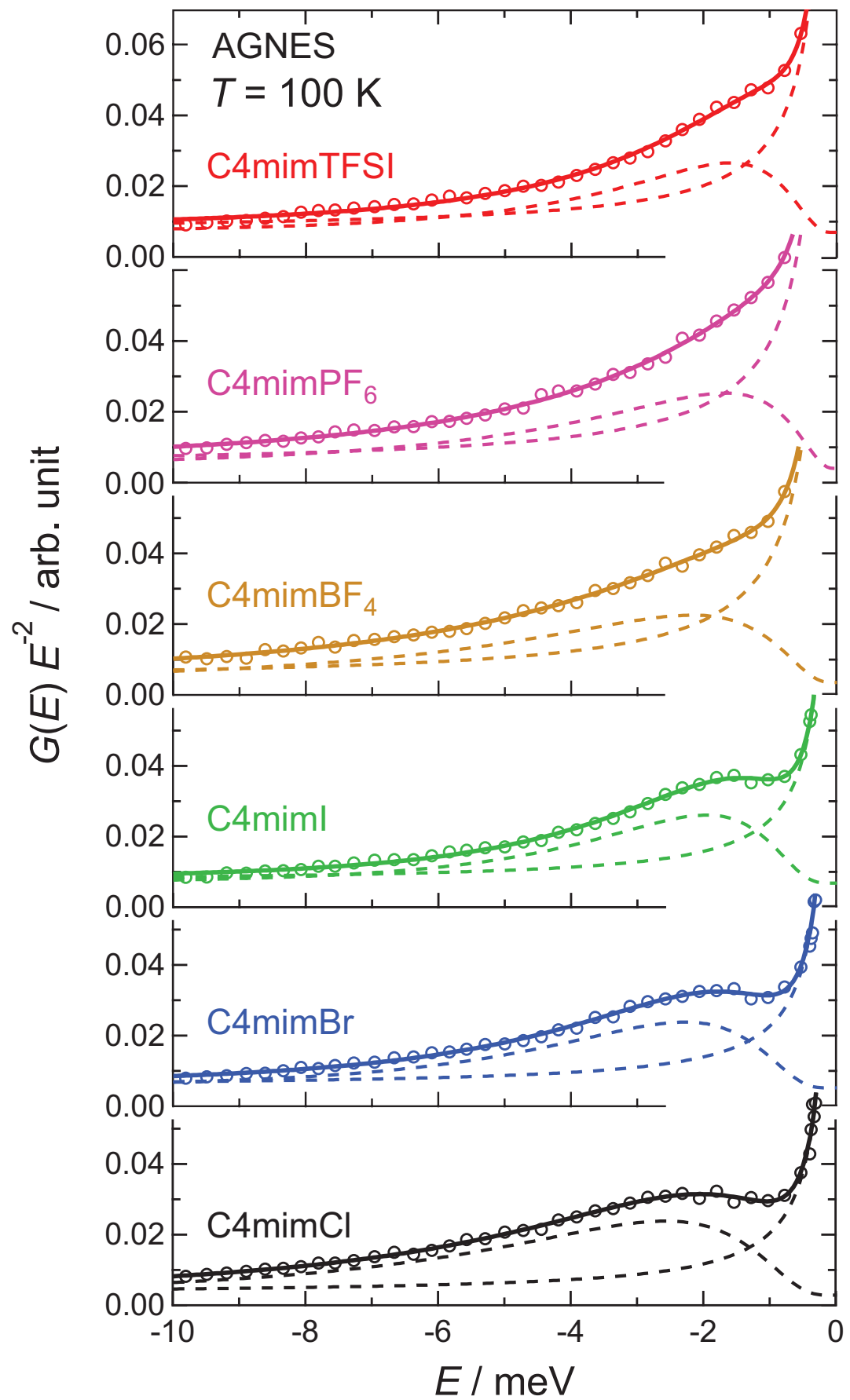

Figure 2: Vibrational density of states devided by $E^{2}, g(E) / E^{2}$, in an arbitrary scale for $\mathrm{C} 4 \mathrm{mimX}\left(\mathrm{X}=\mathrm{Cl}, \mathrm{Br}, \mathrm{I}, \mathrm{BF}_{4}, \mathrm{PF}_{6}\right.$, TFSI) at $T=100 \mathrm{~K}$. Solid and dashed curves represent the results of the fit, which is described in the text. 


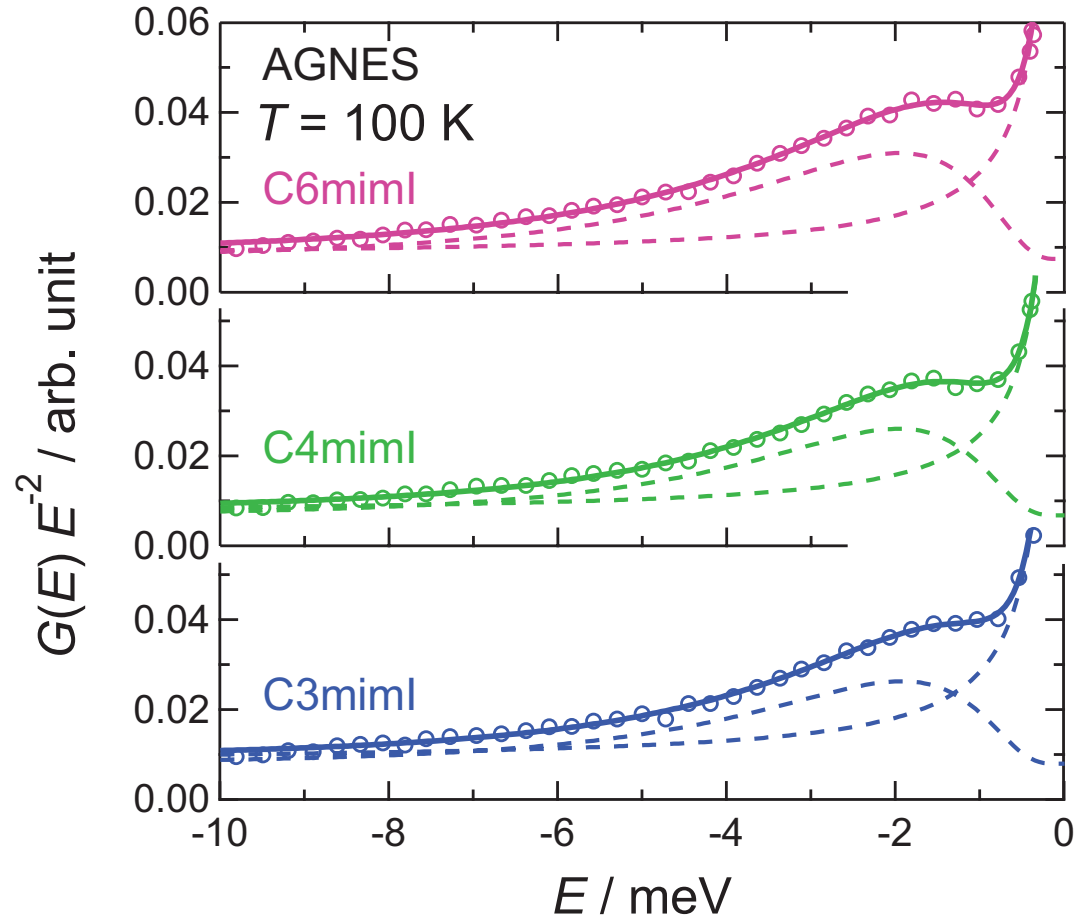

Figure 3: Vibrational density of states devided by $E^{2}, g(E) / E^{2}$, in an arbitrary scale for $\mathrm{C} n \operatorname{minI}(n=3,4,6)$ at $T=100 \mathrm{~K}$. Solid and dashed curves represent the results of the fit, which is described in the text. 


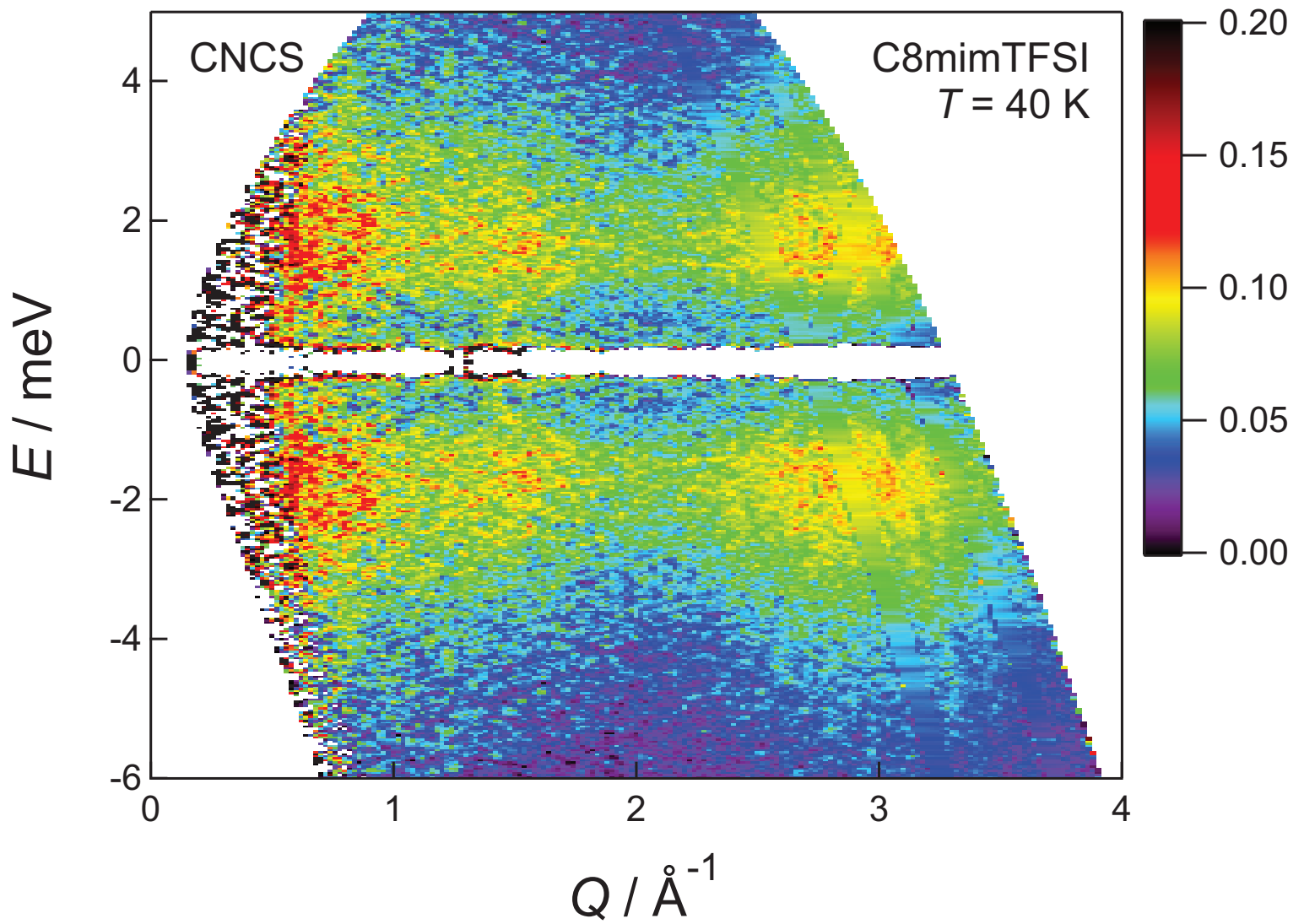

Figure 4: Map of $S(Q, E)_{\text {red }} / Q$ in an arbitrary scale for d-C8mimTFSI measured at $T=40 \mathrm{~K} . S(Q, E)_{\text {red }} / Q$ is defined in the text. The scattering intensity is divided by $Q$ for the sake of clarify. 


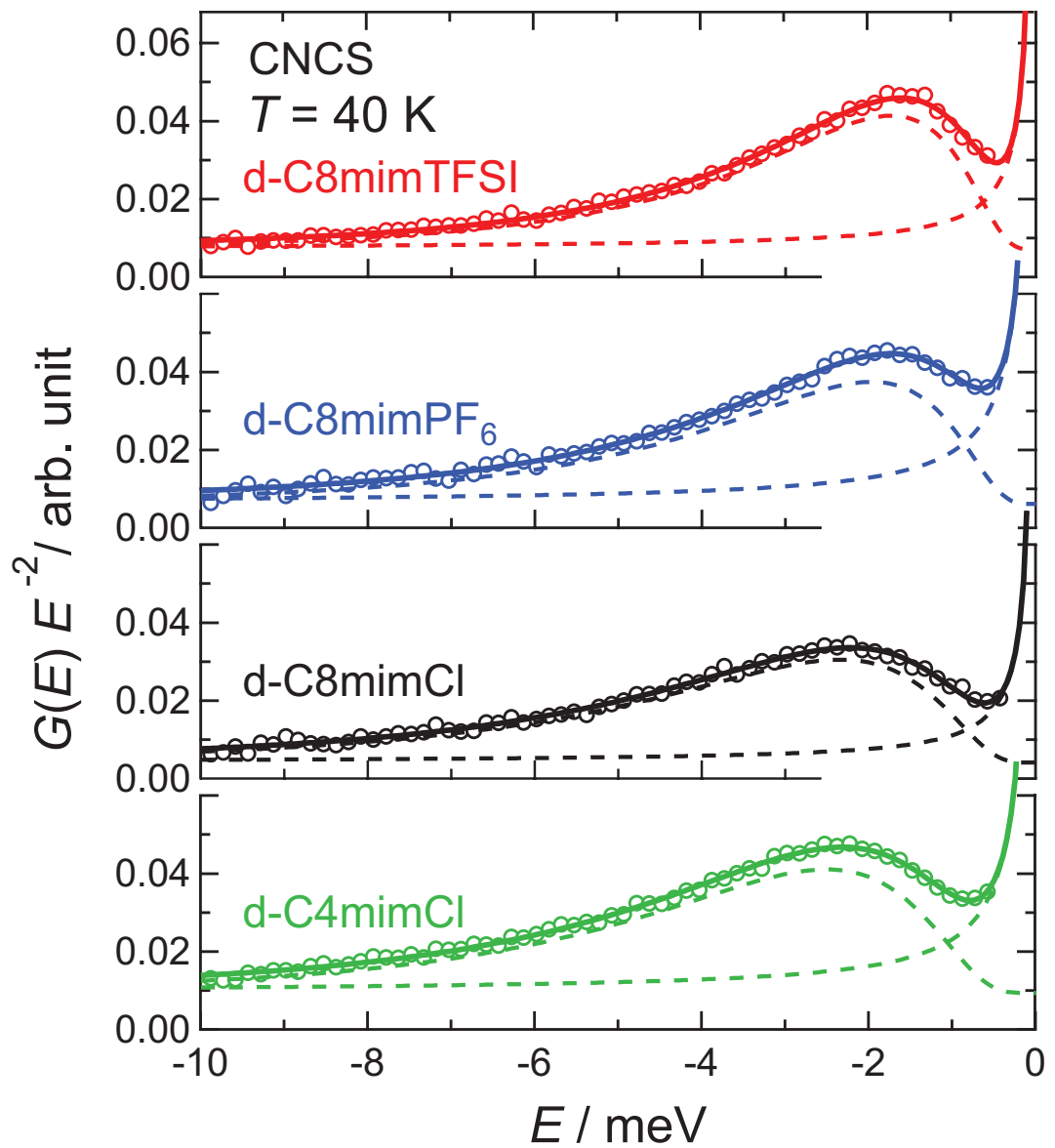

Figure 5: Vibrational density of states devided by $E^{2}, g(E) / E^{2}$, in an arbitrary scale for d-C4mimCl and d-C $8 \operatorname{mimX}(\mathrm{X}=\mathrm{Cl}$, $\mathrm{PF}_{6}$, TFSI) taken at $T=40 \mathrm{~K}$. Solid and dashed curves represent the results of the fit. See the text for details. 


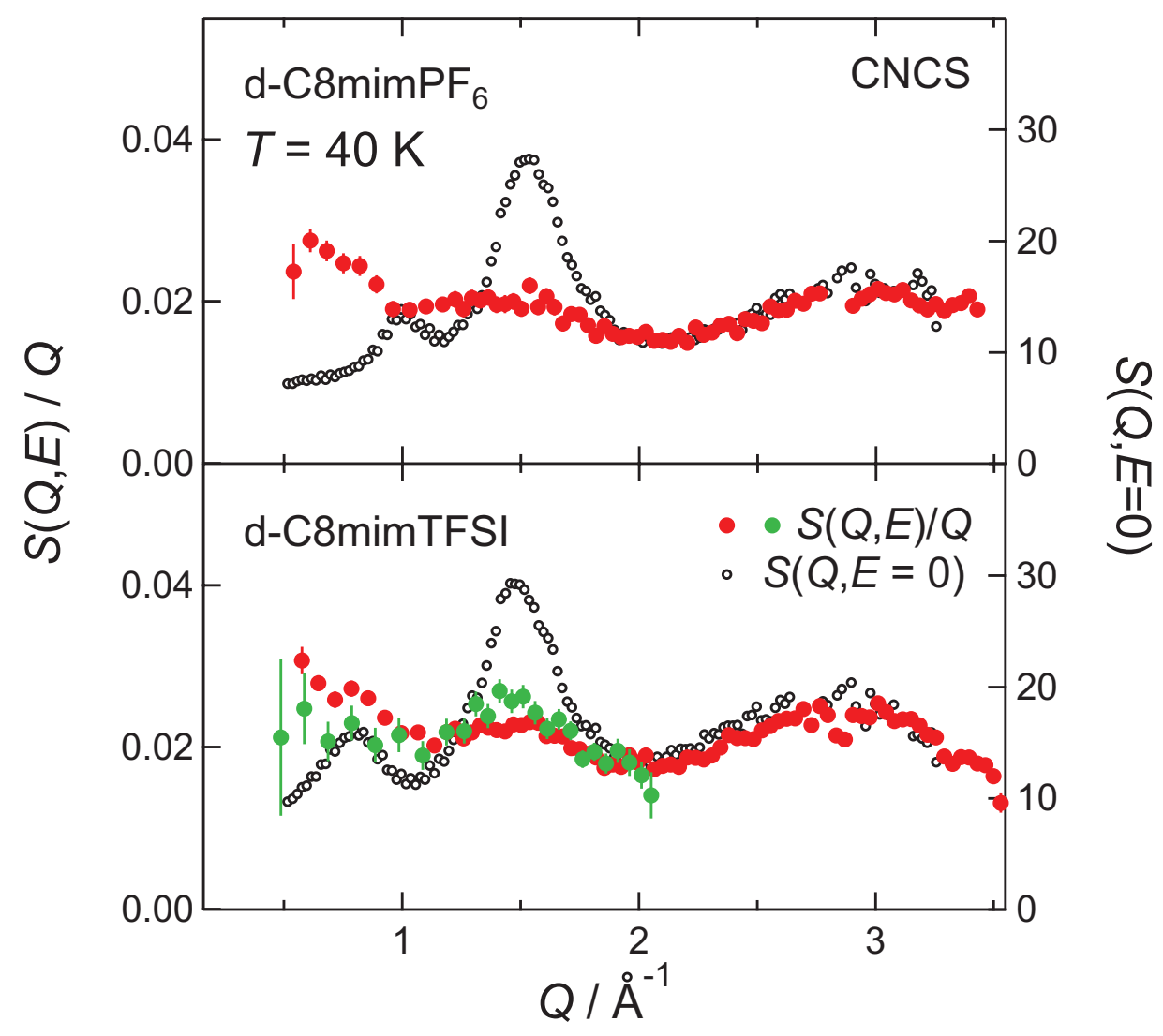

Figure 6: $Q$-dependence of elastic and inelastic scattering intensities for d-C8mimPF 6 and d-C8mimTFSI at $T=40 \mathrm{~K}$. The data are taken on the CNCS spectrometer with the incident energy of $6.6 \mathrm{meV}$. The data with the incident energy of $1.55 \mathrm{meV}$ is also shown for the d-C8mimTFSI sample (green circles). Inelastic and elastic scattering intensities are estimated from the integration over $-2.5<E<-1 \mathrm{meV}$ and $-0.2<E<0.2 \mathrm{meV}$, respectively. The inelastic intensity is divided by $Q$. 


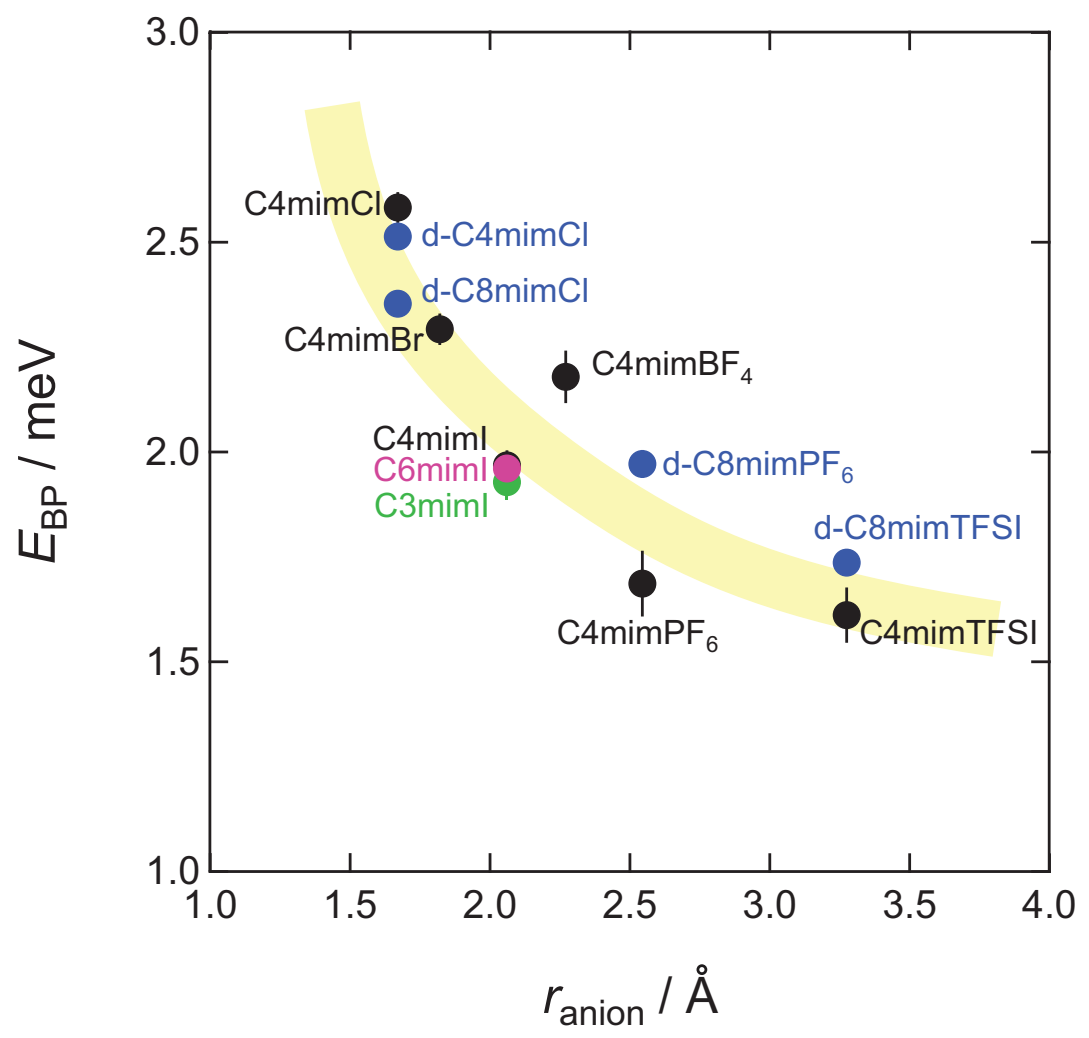

Figure 7: Boson peak energies against anion radii. 


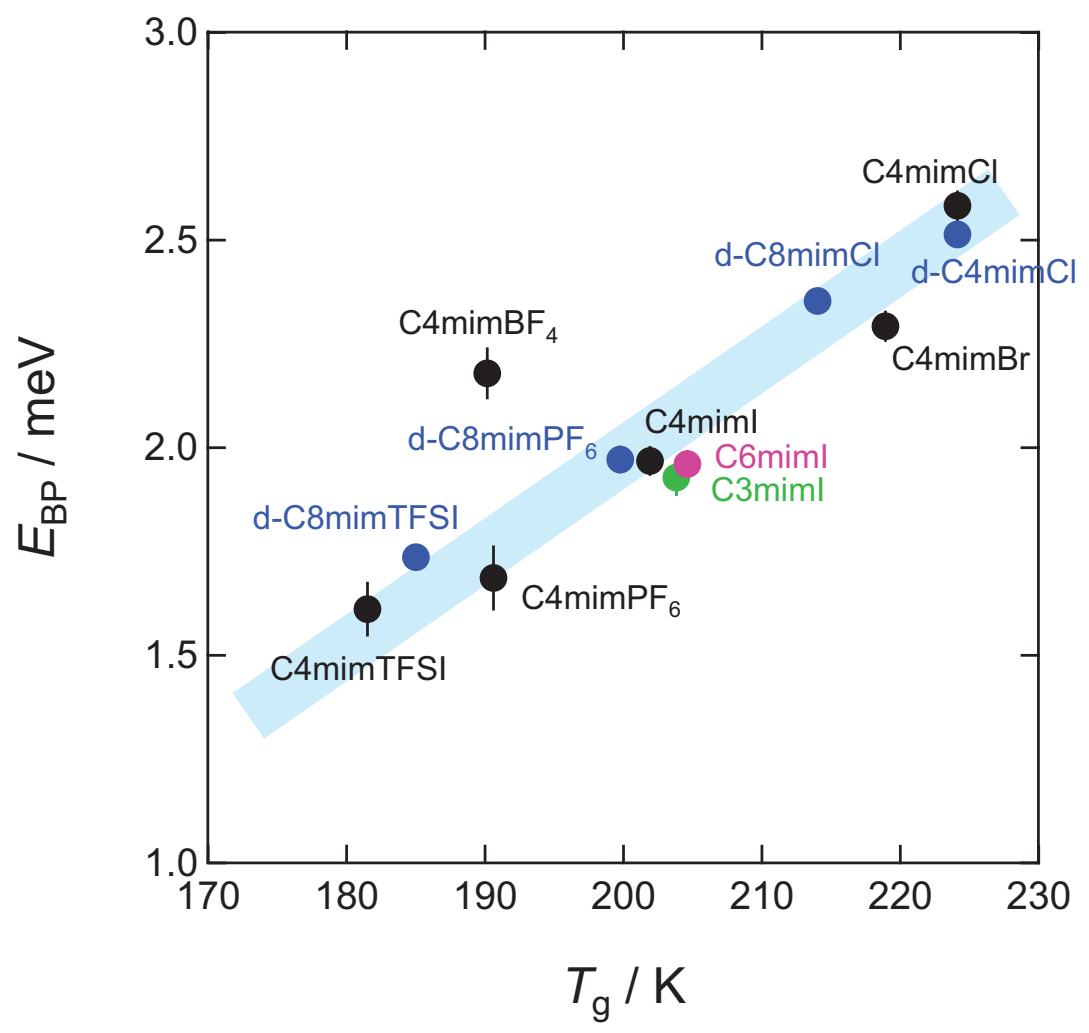

Figure 8: Boson peak energies against glass transition temperatures. 


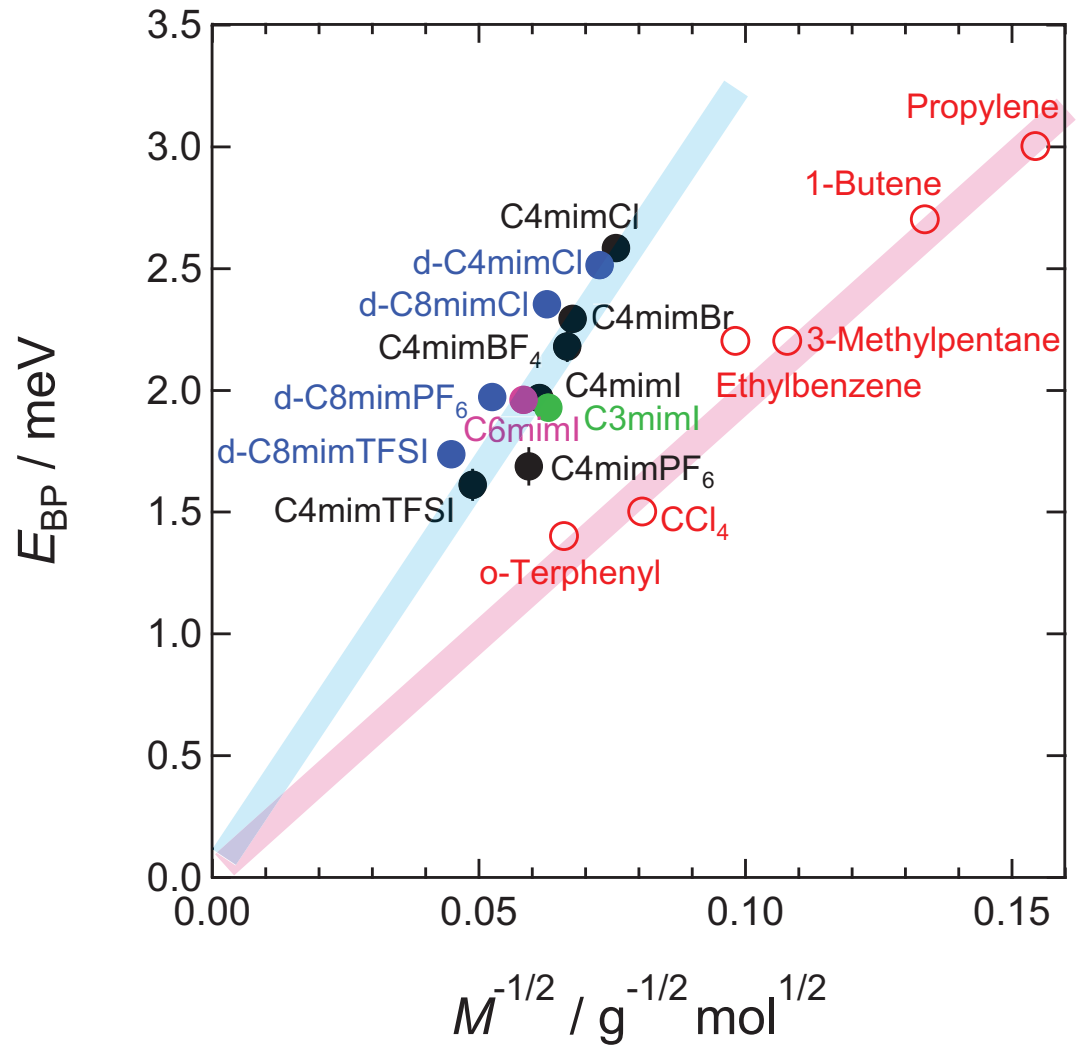

Figure 9: Molecular mass dependence of boson peak energy. The data for some molecular liquids are also shown for comparison. 